\title{
Recent Advances of TIRADS Classification of Thyroid Nodules by Ultrasound
}

Ayman Mohammed Ibrahim, Wessam Sherin Shokry, Hanan Ebrahim Mousa Ebrahim*

Radiodiagnosis Department, Faculty of Medicine, Ain Shams University

*Corresponding author: Hanan Ebrahim Mousa Ebrahim, Mobile: (+20) 01111134983 ,

E-Mail: hananibrahim.010@gmail.com

\begin{abstract}
Background: Thyroid nodules are abnormal growths of thyroid tissue which commonly arise within an otherwise normal thyroid gland. They may be hyperplasia or a thyroid neoplasm, but only a small percentage of the latter are thyroid cancers.

Objectives: To improve the management of the patients and to reduce cost-effectiveness by decreasing number of unnecessary fine needle aspiration cytology (FNAC).

Patients and Methods: This study was conducted on 50 patients known or clinically suspected to have solitary thyroid nodule or multiple nodules, referred to Radiodiagnosis Department, Ain Shams University Hospitals. The cases included 33 females and 17 males (age between 41-50 years).

Results: All the nodules with TIRADS 1 were benign. In TIRADS 2 there were 10 benign and 1 malignant nodules. In TIRADS 3 there were 4 benign and 1 malignant nodules. In TIRADS 4 there were 2 benign nodules and 4 benign nodules while in TIRADS 5 there were 2 benign and 4 malignant nodules. There was a statistically significant difference between the two groups. There was a statistically significant difference between the two groups with benign and malignant nodules regarding the texture of the nodules. Solid texture was higher in the malignant nodules and spongiform texture was common in the benign nodules while the cystic and mixed texture were comparable between the two groups.

Conclusion: The major ultrasound features seen associated with malignancy were microcalcifications, taller than wider shape of the nodule, hypoechoic and marked hypoechoic echopattern of the nodule, irregular borders and presence of suspicious cervical lymph nodes.
\end{abstract}

Keywords: Thyroid Nodules, TIRADS Classification, Ultrasound.

\section{INTRODUCTION}

Thyroid nodules are abnormal growths of thyroid tissue which commonly arise within an otherwise normal thyroid gland. They may be hyperplasia or a thyroid neoplasm, but only a small percentage of the latter are thyroid cancers. Small, asymptomatic nodules are common, and many people who have them are unaware of them. Thyroid nodules can be felt as a lump in the throat. When they are large, they can sometimes be seen as a lump in the front of the neck. Sometimes a thyroid nodule presents as a fluid-filled cavity called a thyroid cyst. Often, solid components are mixed with the fluid ${ }^{(\mathbf{1})}$.

Inflammation of the thyroid is called thyroiditis. Inflamed thyroids may cause symptoms of hyperthyroidism or hypothyroidism. Two types thyroiditis initially present with hyperthyroidism and are sometimes followed by a period of hypothyroidism; Hashimoto's thyroiditis and postpartum thyroiditis. There are other disorders that cause inflammation of the thyroid, and these include subacute thyroiditis, acute thyroiditis, silent thyroiditis, Riedel's thyroiditis and traumatic injury, including palpation thyroiditis ${ }^{(2)}$.

Ultrasound of the thyroid may be used to reveal whether structures are solid or filled with fluid, helping to differentiate between nodules and goitres and cysts. It may also help differentiate between malignant and benign lesions. A fine needle aspiration biopsy may be taken concurrently of thyroid tissue to determine the nature of a lesion. This can determine the size and shape of lesions, reveal whether nodules or goitres are metabolically active, and reveal and monitor sites of thyroid disease or cancer deposits outside the thyroid ${ }^{(3)}$.

Ultrasound imaging is useful as the firstline, non-invasive investigation in determining the size, texture, position, and vascularity of a nodule, accessing lymph nodes metastasis in the neck, and for guiding fine needle aspiration cytology (FNAC) or biopsy. Ultrasonographic findings will also guide the indication to biopsy and the long term follow-up ${ }^{(4)}$.

After a nodule is found during a physical examination, most commonly an ultrasound is performed to confirm the presence of a nodule, and assess the status of the whole gland. Measurement of thyroid stimulating hormone and anti-thyroid antibodies will help decide if there is a functional thyroid disease such as Hashimoto's thyroiditis present, a known cause of a benign nodular goitre. Fine needle biopsy for histopathology is also used (5).

There is a high prevalence of thyroid nodules on ultrasonographic (US) examination. However, most of them are benign. US criteria may help to decide cost-effective management. The 
TIRADS has allowed us to improve patient management and cost-effectiveness, avoiding unnecessary FNAB. In addition, we have established standard codes to be used both for radiologists and endocrinologists. Thyroid nodules are highly prevalent; about one third of the adult population has thyroid nodules on ultrasonographic (US) examination ${ }^{(6)}$.

The aim of this work was to develop a practical thyroid imaging reporting and data system (TIRADS) with which to categorize thyroid nodules and stratify their malignant risk.

\section{PATIENTS AND METHODS}

This study was conducted on 50 patients known or clinically suspected to have solitary thyroid nodule or multiple nodules, referred to Radiodiagnosis Department, Ain Shams University Hospitals. The cases included 33 females and 17 males (age between 41-50 years).

\section{Ethical approval:}

An approval of the study was obtained from Ain Shams University academic and ethical committee. Every patient signed an informed written consent for acceptance of the operation.

Inclusion criteria: Patients known or clinically suspected to have solitary thyroid nodule or multiple nodules

Exclusion criteria: We excluded thyrotoxic patients and patients who were unfit for examination.

All patients in this study were subjected to:

1. Complete history taking.

2. Full clinical examination.

3. Ultrasonography and color Doppler evaluation.

\section{Ultrasonographic Technique:}

Ultrasonography and Doppler evaluation of the thyroid lesions were performed with a 7.5-10 $\mathrm{MHz}$ high frequency linear array transducer (Siemens Acuson X300 PE ultrasound systems, German). All images were examined on real-time two-dimensional gray-scale and Doppler imaging. Both lobes of the thyroid gland including the isthmus were evaluated. Patients were scanned in the supine position with the neck mildly hyperextended by an "oatmeal" pillow.

The neck was scanned in sagittal, transverse, and oblique sections to optimally visualize both lobs of thyroid, isthmus, carotid arteries, as well as internal jugular veins. Imaging of lower poles of thyroid was improved by making the patient swallow. This tended to raise the thyroid gland in the neck. The region of the carotid artery and jugular veins laterally and supraclavicular fossa were also examined for any lymphadenopathy.

Nodule classification by ultrasound:

All thyroid nodules were characterized according to the internal component (solid, mixed or cystic). Margins were classified as regular, lobulated or irregular. Echogenicity was classified as hyperechogenicity, isoechogenicity, hypoechogenicity and marked hypoechogenicity. Isoechogenicity was defined as an echogenicity similar to that of the adjacent healthy thyroid gland. A nodule was classified as "marked hypoechogenicity" if the echogenicity was less than that of the superficial surrounding neck muscles.

\section{Ultrasound guided fine needle aspiration (FNA) technique:}

Patient Positioning and Preparation: The patient was placed in a supine position with the neck slightly extended and the skin was cleansed with betadine solution. The 7.5-10 $\mathrm{MHz}$ linear transducer was also sterilized with the same solution. Sterile gel was used as a coupling agent. The needle was inserted through the skin of thyroid region in front of the neck at an oblique angle within the image plane of transducer.

Local Anesthesia: A 2\% lidocaine solution was injected into the skin and superficial subcutaneous tissue at the predetermined site.

Obtaining the Specimen: A 20 gauge needle was used with an attached $10 \mathrm{ml}$ syringe. The transducer was placed directly over the lesion. Before aspiration, scanning was performed in the transverse plane for lesion localization, followed by color Doppler mapping to depict any large blood vessels in and around the nodule so that vascular injury could be avoided during the procedure. The patient was instructed not to swallow or speak during the insertion of the needle. The syringe attached to the needle was placed just below or beside the transducer so that the needle was introduced perpendicular or parallel to the transducer according to the place of the needle below or beside the transducer and the needle tip was carefully monitored during the procedure. When the needle reached the target, the biopsy was taken. The needle tip was advanced into the nodule and vigorously moved to and fro while being rotated on its axis until a small amount of cellular material was collected inside the needle hub. During the procedure, all needle movements were continuously visualized in real time. The collected material was placed on glass slides, smeared, and fixed in 95\% ethyl alcohol. The syringe was rinsed with normal saline solution to obtain any remaining material for use in cell blocking. 
Post-procedural Care: After the procedure, plaster was applied, and the patient was instructed to manually compress the skin entry site for a minimum of 15 minutes.

\section{Statistical analysis}

Recorded data were analyzed using the statistical package for the social sciences, version 20.0 (SPSS Inc., Chicago, Illinois, USA). Quantitative data were expressed as mean \pm standard deviation (SD), median, and interquartile range (IQR) and were compared by Mann-Whitney (z) test. Qualitative data were expressed as frequency and percentage and were compared by $\mathrm{Chi}^{2}\left(\chi^{2}\right)$ test. So, the $p$-value was considered significant as the following: P-value $\leq 0.05$ was considered significant, $\mathrm{P}$-value $\leq 0.001$ was considered as highly significant, $\mathrm{P}$-value $>0.05$ was considered insignificant.

\section{RESULTS}

Table 1: Demographic data in the cases of the study.

\begin{tabular}{|l|c|}
\hline \multicolumn{1}{|c|}{ Items } & $\begin{array}{c}\text { Study cases } \\
\mathbf{N}=\mathbf{5 0}\end{array}$ \\
\hline Sex & $\mathbf{1 3}(\mathbf{2 6 \%})$ \\
\hline Male & $\mathbf{3 7}(\mathbf{7 4 \%})$ \\
\hline Female & $\mathbf{4 4 . 2 4 \pm 8 . 8 5}$ \\
\hline Age (years) & $\mathbf{4 3 . 5}(\mathbf{2 9 - 6 8 )}$ \\
\hline Mean \pm SD & $\mathbf{2 ( 4 \% )}$ \\
\hline Median (range) & $\mathbf{1 5}(\mathbf{3 0 \%})$ \\
\hline Age group & $\mathbf{2 0}(\mathbf{4 0 \%})$ \\
\hline $21-30$ & $\mathbf{9 ( 1 8 \% )}$ \\
\hline $31-40$ & $\mathbf{4 ( 8 \% )}$ \\
\hline $41-50$ &
\end{tabular}

Continuous data expressed as mean $\pm \mathrm{SD}$ and median (range)

Categorical data expressed as Number (\%)

Table (2) shows that all the nodules with TIRADS 1 were benign. There was a statistically significant difference between the two groups.

Table 2: Relation between pathological type and TIRADs.

\begin{tabular}{|c|c|c|c|c|}
\hline \multirow{2}{*}{ TIRADs } & \multicolumn{2}{|c|}{ Nature } & \multirow{2}{*}{$\begin{array}{c}\text { Risk of } \\
\text { malignancy }\end{array}$} & $\begin{array}{c}\text { Test of } \\
\text { significance }\end{array}$ \\
\cline { 2 - 3 } & Benign (n=40) & Malignant (n=10) & \multirow{2}{*}{$\chi^{2}=20.271$} \\
\hline 1 & $21(52.5 \%)$ & $0(0 \%)$ & $0 \%$ & \multirow{2}{*}{$\mathrm{P}<0.001 *$} \\
\hline 2 & $10(25 \%)$ & $1(10 \%)$ & $9.1 \%$ & \\
\hline 4 & $4(10 \%)$ & $1(10 \%)$ & $20 \%$ & \\
\hline 5 & $2(5 \%)$ & $4(40 \%)$ & $66.7 \%$ & $57.1 \%$ \\
\hline
\end{tabular}

*: significant value

Table (3) revealed that the size of the benign nodules was statistically significantly larger as compared with the malignant nodules.

Table 3: Comparison of size of the nodules according to nature of the lesions.

\begin{tabular}{|c|c|c|c|}
\hline & Benign $(n=58)$ & Malignant $(n=22)$ & Test of significance \\
\hline \multicolumn{4}{|c|}{ Size of nodules (mm) } \\
\hline Mean \pm SD & $28.10 \pm 8.84$ & $18.20 \pm 9.24$ & $z=-2.841$ \\
\hline Median (range) & $28(22-36.5)$ & $17(10.5--23.25)$ & $\mathrm{p}=0.004 *$ \\
\hline
\end{tabular}

Continuous data expressed as median (IQR) and mean \pm SD; *: statistically significant

As shown in table (4), there was a statistically significant difference between the two groups with benign and malignant nodules regarding the texture of the nodules. Solid texture was higher in the malignant nodules and spongiform texture was common in the benign nodules while the cystic and mixed texture were comparable between the two groups. 
Table 4: Relation between nature of the lesions and texture.

\begin{tabular}{|c|c|c|c|}
\hline \multirow{2}{*}{ Texture } & \multicolumn{2}{|c|}{ Nature } & \multirow{2}{*}{$\begin{array}{c}\text { Test of } \\
\text { significance }\end{array}$} \\
\cline { 2 - 3 } & $\begin{array}{c}\text { Benign } \\
(\mathbf{n = 4 0 )}\end{array}$ & $\begin{array}{c}\text { Malignant } \\
(\mathbf{n = 1 0})\end{array}$ & \multirow{2}{*}{$\chi^{2}=13.522$} \\
\hline Cystic & $17(42.5 \%)$ & $4(40 \%)$ & $1(10 \%)$ \\
\hline Mixed & $6(15 \%)$ & $4(40 \%)$ & $\mathrm{P}<0.004 *$ \\
\hline Solid & $1(2.5 \%)$ & $1(10 \%)$ & \\
\hline Spongiform & $16(40 \%)$ & & \\
\hline
\end{tabular}

Categorical data expressed as Number (\%); *: significant value

As shown in table (5), there was no statistically significant difference between the two groups with benign and malignant nodules regarding the echogenicity of the nodules. Hypoechoic nodules were higher in the malignant nodules.

Table 5: Relation between nature of the lesions and echogenicity.

\begin{tabular}{|c|c|c|c|}
\hline \multirow{2}{*}{ Echogenicity } & \multicolumn{2}{|c|}{ Nature } & \multirow{2}{*}{ Test of significance } \\
\cline { 2 - 3 } & Benign $(\mathbf{n}=\mathbf{4 0})$ & Malignant $(\mathbf{n}=\mathbf{1 0})$ & \\
\\
\hline Anechoic & $13(32.5 \%)$ & $4(40 \%)$ & \multirow{2}{*}{$\chi^{2}=8.527$} \\
\hline Hyperechoic & $14(35 \%)$ & $0(0 \%)$ & \multirow{2}{*}{$=0.074$} \\
\hline Hypoechoic & $8(20 \%)$ & $5(50 \%)$ & \\
\hline Marked hypoechoic & $1(2.5 \%)$ & $1(10 \%)$ & \\
\hline Isoechoic & $4(10 \%)$ & $0(0 \%)$ & \\
\hline
\end{tabular}

Categorical data expressed as Number (\%); *: significant value

As shown in table (6), there was a statistically significant difference between the two groups with benign and malignant nodules regarding the echogenic foci.

Table 6: Relation between nature of the lesions and echogenic foci.

\begin{tabular}{|c|c|c|c|}
\hline \multirow{2}{*}{ Echogenic foci } & \multicolumn{2}{|c|}{ Nature } & \multirow{2}{*}{ Test of significance } \\
\cline { 2 - 3 } & $\begin{array}{c}\text { Benign } \\
(\mathbf{n = 4 0 )}\end{array}$ & $\begin{array}{c}\text { Malignant } \\
(\mathbf{n = 1 0})\end{array}$ & \multirow{2}{*}{$\begin{array}{c}\chi^{2}=17.532 \\
\mathrm{P}<0.001^{*}\end{array}$} \\
\hline Macro calcification & $10(25 \%)$ & $1(10 \%)$ & $5(50 \%)$ \\
\hline None & $30(75 \%)$ & $3(30 \%)$ & \\
\hline Peripheral & $0(0 \%)$ & $1(10 \%)$ & \\
\hline Punctuate & $0(0 \%)$ & & \\
\hline
\end{tabular}

Categorical data expressed as Number (\%); *: significant value

As shown in table (7), there was a statistically significant difference between the two groups with benign and malignant nodules regarding the halo sign within the nodules.

Table 7: Relation between nature of the lesions and halo sign.

\begin{tabular}{|c|c|c|c|}
\hline \multirow{2}{*}{ Halo sign } & \multicolumn{2}{|c|}{ Nature } & \multirow{2}{*}{ Test of significance } \\
\cline { 2 - 3 } & Benign (n=40) & Malignant (n=10) & \\
\hline Absent & $4(10 \%)$ & $4(40 \%)$ & $\chi^{2}=5.357$ \\
$\mathrm{P}<0.021^{*}$
\end{tabular}

Categorical data expressed as Number (\%); *: significant value

As shown in table (8), there was a statistically significant difference between the two groups with benign and malignant nodules regarding the vascularity shape. Taller than wider nodules were more common in the malignant lesions.

Table 8: Relation between nature of the lesions and vascularity shape.

\begin{tabular}{|c|c|c|c|}
\hline \multirow{2}{*}{ Vascularity shape } & \multicolumn{2}{|c|}{ Nature } & \multirow{2}{*}{ Test of significance } \\
\cline { 2 - 3 } & Benign $(\mathbf{n}=\mathbf{4 0})$ & Malignant $(\mathbf{n}=\mathbf{1 0})$ & \\
\hline Taller than wider & $3(7.5 \%)$ & $3(30 \%)$ & $\chi^{2}=3.858$ \\
\hline Wider than taller & $37(92.5 \%)$ & $7(70 \%)$ & $\mathrm{P}=0.052$ \\
\hline
\end{tabular}

Categorical data expressed as Number (\%); *: significant value 


\section{ILLUSTRATED CASES}

\section{CASE (1)}

Female patient aged 51 years presented with neck swelling. Neck ultrasound image of the right lobe shows rounded measures $2.9 \times 2.7 \mathrm{~cm}$, well defined margin hyperechoic solid nodule with central cystic area. Color Doppler: showed intranodular vascularity. TIRADS category: T3. Diagnosis: suspicious for follicular neoplasm for FNAC.

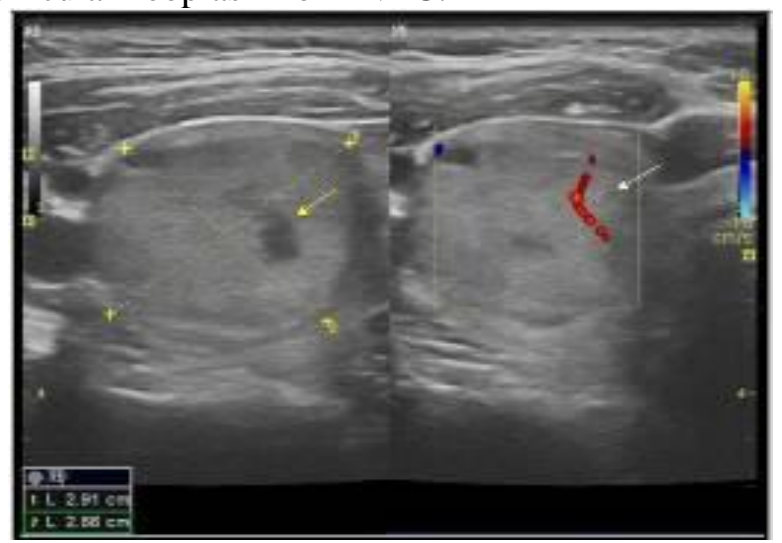

Figure 1: Solid hyperechoic nodule with cystic area (yellow arrow) and intranodular vascularity (white arrow).

\section{CASE (2)}

Female patient aged 60 years presented with left neck swelling. Neck ultrasound image of the left lobe shows rounded measures $1.3 \times 1.3 \mathrm{~cm}$, well defined margin markedly hypoechoic solid nodule with macrocalcification. Color Doppler: showed intranodular vascularity. TIRADS category: T4B. Diagnosis: suspicious for malignancy (medullary carcinoma).

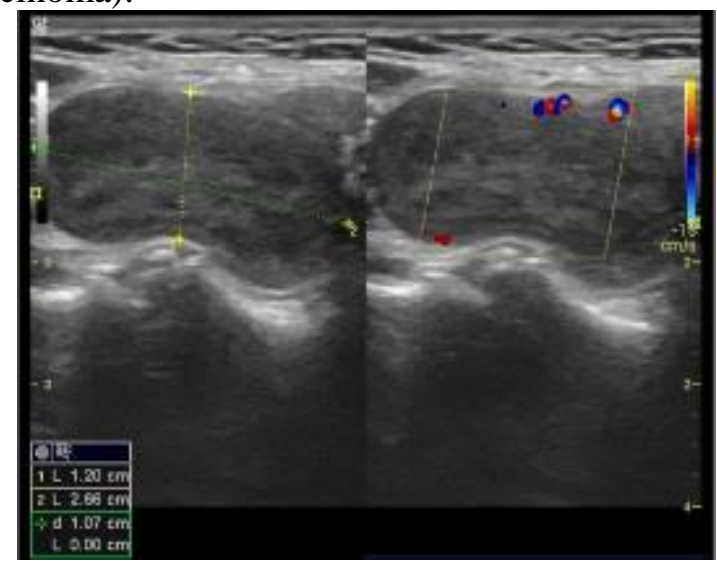

Figure 2: Markedly hypoechoic nodule, macrocalcification (red arrow) and intranodular vascularity (yellow arrow).

\section{CASE (3)}

Male patient aged 60 years old presented with neck swelling. Neck ultrasound image of the left lobe shows taller than wide measure $1.9 \times 2 \mathrm{~cm}$, irregular margin, hypoechoic solid nodule with macrocalcification. Suspicious cervical Lymph node is detected, showed necrotic changes and internal soft tissue nodule, color Doppler: showed intranodular vascularity. TIRADS category: T5, Diagnosis: (suspicious for malignancy) for FNAC.
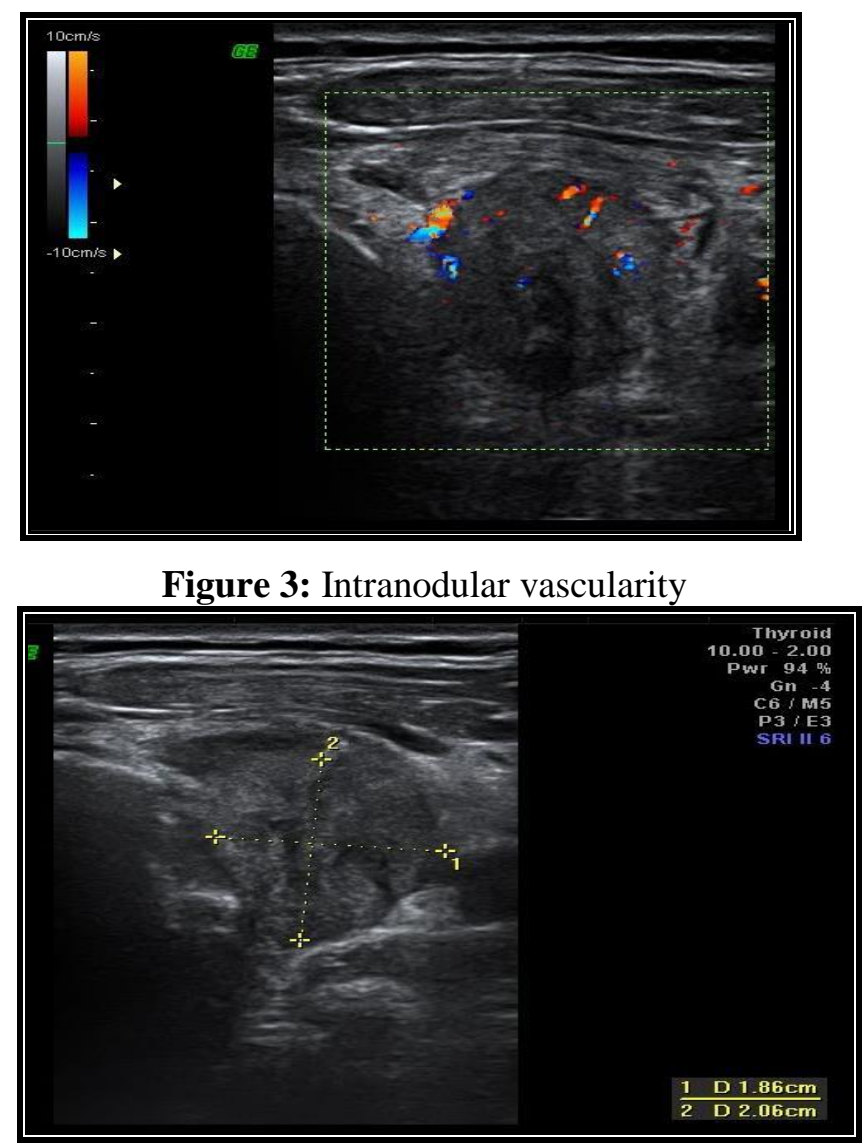

Figure 4: Taller than wide hypoechoic nodule

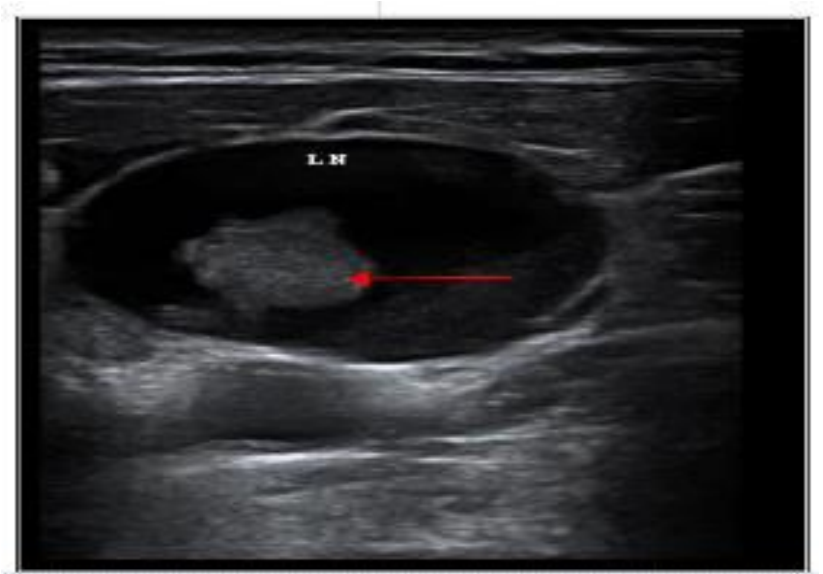

Figure 5: Necrotic changes in cervical lymph node, internal soft tissue nodule (red arrow)

\section{DISCUSSION}

The study included 50 cases who suffered from thyroid gland enlargement or with disturbance in thyroid hormones level. There were 13 males (26\%) and 37 females (74\%) with mean age of 
$44.24 \pm 8.85$ years with range between 29 and 68 years. Most of the cases were in the age group between 41 and 50 years $(40 \%)$ followed by age range between 31 and 40 years $(30 \%)$. In a recent Egyptian study, a total of 40 patients was included in the study, seven males (17.5\%) and 33 females $(82.5 \%)$. The mean patient age was $44.48 \pm 11.04$ years old (range 25-69 years) ${ }^{(7)}$. Horvath et al. ${ }^{(8)}$ included total of 502 nodules (in 210 patients) and out of the total number of patients, 164 were women (78.1 \% of the sample group). In another study, thyroid ultrasound was performed in 450 patients (350 females and 100 males), and their age ranged between 10 and 70 years with a mean of $38.7 \pm 15.7$ years ${ }^{(9)}$. Dawoud and Dawoud ${ }^{(10)}$ included 60 patients (37 females and 23 male) with solitary thyroid nodule, their age ranged from 21 to 52 years (mean age 30.6 years). As reported in all the previous results including the results of this current study, thyroid nodules is more common in females however, the risk of malignancy of the thyroid nodules is more common in male gender ${ }^{(\mathbf{1 1})}$.

In this study, 21 cases (42\%) had TIRADS 1 score, 11 cases (22\%) had TIRADS 2 score, 5 cases (10\%) had TIRADS 3 score, 6 cases (12\%) had TIRADS 4 score and 7 cases (14\%) had TIRADS 5 score. In the study conducted by Horvath et $\boldsymbol{a l} .{ }^{(8)}$ the overall distribution in TIRADS categories was as follows: 116 TIRADS 2 (23.11\%), 56 TIRADS 3 $(11.15 \%), 243$ TIRADS $4(48.41 \%)$, and 87 TIRADS $5(17.33 \%)$. In another study, the overall distribution in the TIRADS and American Thyroid Association (ATA) categories was as follows: 5 TR2 (3.6\%), 43 TR3 (30.7\%), 32 TR4a (22.9\%), 9 TR4b (6.4\%), 23 TR4c (16.4\%), 28 TR5 (20.0\%) ${ }^{(12)}$. The distribution of thyroid nodules by TIRADS categories in the study conducted by Ashamallah and ELAdalany $^{\left({ }^{9}\right)}$ was 80 cases $(17.7 \%$ ) in TIRADS 1,70 $(15.5 \%)$ in TIRADS 2, $110(24.4 \%)$ in TIRADS 3, 36 $(8 \%)$ in TIRADS 4A, $52(11.5 \%)$ in TIRADS 4B and $102(22.6 \%)$ in TIRADS 5.

In this study, the risk of malignancy was $0 \%$ for TIRADS $1,9.1 \%$ for TIRADS $2,20 \%$ for TIRADS $3,66.7 \%$ for TIRADS 4 and $57.1 \%$ for TIRADS 5. This came in accordance with Horvath $\boldsymbol{e t}$ al. ${ }^{(8)}$ who showed that the percentage of malignancy for each category was as follows: $0 \%(0 / 116)$ in TIRADS $2,1.79 \%(1 / 56)$ in TIRADS $3,76.13 \%$ (185/243) in TIRADS 4 [considering subgroups: 5.88 $\%(1 / 17)$ in TIRADS 4A, $62.82 \%$ (49/78)in TIRADS 4B, $91.22 \%(135 / 148)$ in TIRADS 4C] , and 98.85\% (86/87) in TIRADS 5. This is comparable to Paschke et $\boldsymbol{~ a l}^{\left({ }^{(13)}\right.}$ as they estimated a risk of malignancy of $0 \%$ in TIRADS $2,3.4 \%$ in TIRADS $3,10-80 \%$ in TIRADS 4, and $87 \%$ in TIRADS 5. Periakaruppan et al. ${ }^{(14)}$ described that a malignancy risk of $0 \%$ is expected for TIRADS $2,1.7 \%$ for TIRADS 3 , a risk of $3.3-72.4 \%$ for TIRADS 4 , and of $87.5 \%$ for TIRADS 5. In the study conducted by Moifo et al. ${ }^{(15)}, 430$ nodules were assessed. Twenty-three nodules out of these 430 nodules (5.3\%) were malignant. The malignancy risk of the TIRADS categories were $0 \%$ for TIRADS2, $2.2 \%$ for TIRADS3, 5.9-57.9\% for TIRADS4, and $100 \%$ for TIRADS5. Chandramohan et $\boldsymbol{a l}$. ${ }^{(16)}$ assessed the practical aspects and accuracy of TIRADS in daily clinical practice observed that positive predictive value (PPV) for malignancy was $6.6,32,36,64,59$, and $91 \%$ for TIRADS 2, 3, 4A, 4B, 4C, and 5 categories. According to another similar study from Indian literature by Srinivas et al. ${ }^{(17)}$, it was concluded that the risk of malignancy for TIRADS categories 1, 2, 3, 4A, 4B, 4C, and 5 was $0,0,0.64,4.76,66.67,83.33$, and $100 \%$, respectively. The risk of malignancy calculated by the OR $(95 \% \mathrm{CI})$ is 0 for TIRADS 1 and 2, 0.2 for TIRADS 3, 0.67 for TIRADS 4A, 2 for

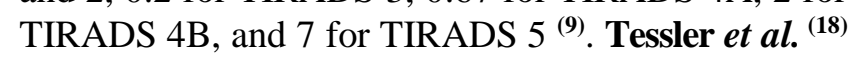
found an increased risk of malignancy in thyroid nodules starting from TR3 (5\%) to TR4 and TR5 (20\%). Another study done by Middleton et al. ${ }^{(19)}$ stated that the risk of malignancy is $4.8 \%, 9.1 \%$, and $35 \%$, for TR 3, TR4, and TR5 respectively.

In this study, all the nodules with TIRADS 1 were benign. In TIRADS 2 there were 10 benign and 1 malignant nodules. In TIRADS 3 there were 4 benign and 1 malignant nodules. In TIRADS 4 there were 2 benign nodules and 4 benign nodules while in TIRADS 5 there were 2 benign and 4 malignant nodules. There was a statistically significant difference between the two groups. This came in agreement with Azab et al. ${ }^{(7)}$ who showed that the best cutoff point to detect malignant cases was five which corresponds to TR4 with a sensitivity of $88.89 \%$, specificity $96.77 \%$, positive predictive value (PPV) of $88.9 \%$, and negative predictive value (NPP) of $69.8 \%$. According to the results of Ashamallah and EL-Adalany ${ }^{(\mathbf{9})}$, sensitivity was 0\% TIRADS (1 and 2), 9.1\% TIRADS (3), 25\% TIRADS (4A), 60\% TIRADS(4B), $100 \%$ TIRADS (5). Specificity was $59.5 \%$ TIRADS (1), $60.5 \%$ TIRADS (2), $58.8 \%$ TIRADS (3), 65.9\% TIRADS (4A), 70\% TIRADS (4B), 85.7\% TIRADS (5). In this last study the PPV of malignancy increases from $0 \%$ for TIRADS type 1 and type 2 to $6.7 \%$ for TIRADS type 3 and type 4A to $20 \%$ for TIRADS type 4B to reach $67 \%$ for TIRADS type 5 . In this study the NPV was $73.3 \%$ for TIRADS $1,76.6 \%$ for TIRADS 2, 66.7\% for TIRADS 3, $90 \%$ for TIRADS 4A, $93.3 \%$ for TIRADS 4B and $100 \%$ for TIRADS 5. Russ et al. ${ }^{(\mathbf{2 0})}$ stated that PPV for each score was $0 \%$ for type $2,0.25 \%$ for type 3 , $6 \%$ for type $4 \mathrm{~A}, 69 \%$ for type $4 \mathrm{~B}$ and $100 \%$ for type 5. The sensitivity, specificity, negative predictive 
value, positive predictive value and accuracy of the overall TIRADS score were $95.7 \%, 61 \%, 99.7 \%$ and $62 \%$.

In this study, there was a statistically significant difference between the two groups with benign and malignant nodules regarding the texture of the nodules. Solid texture was higher in the malignant nodules and spongiform texture was common in the benign nodules while the cystic and mixed texture were comparable between the two groups. This came in agreement with Azab et al. ${ }^{(7)}$ who showed that all nodules with cystic changes (predominantly cystic) were benign, and there was a highly significant increase in the incidence of solid nodules in the malignant group than in the benign group. Malignancy is uncommon with predominantly cystic nodules ${ }^{(19)}$. Also, Ahn et al. ${ }^{(21)}$ found that thyroid cancers are more likely to be solid or nearly entirely solid.

Regarding echogenicity, there were many studies that showed that the risk of malignancy is inversely proportional to nodule echogenicity; the more echogenicity, the less possibility of malignancy. Papillary and medullary thyroid cancer appears hypoechoic due to increased cellular impaction ${ }^{(22)}$. The exception to this theory is the follicular dominant pathology which is composed of small microfollicles and tends to appear hyperechoic ${ }^{(23,24)}$.

In this study, hypoechoic nodules were higher in the malignant nodules, however, there was no statistically significant difference between the two groups with benign and malignant nodules regarding the echogenicity of the nodules. In the study by Dawoud and Dawoud ${ }^{(\mathbf{1 0})}$, nodule hypoechogenicity was found in $15(88.2 \%)$ of 17 malignant nodules and false positive results were found in 15 nodules, no hyperechogenicity detected in malignant thyroid nodules. Nodule hypoechogenicity used alone as US patterns predictive of malignancy had sensitivity $88.24 \%$, specificity $65.12 \%$ and accuracy $71.67 \%$. Azab et al. ${ }^{(7)}$ showed that there was a highly significant increase in anechoic and hyperechoic nodules in the benign group $(\mathrm{P}$ value $=0.025$ and 0.036 respectively), while there was high increased incidence of very hypoechoic nodules in the malignant group $(\mathrm{P}$ value $=0.001)$ and there was no significant difference between the benign and the malignant groups regarding isoechoic and hypoechoic thyroid nodules; this could be related to the histological subtypes of neoplasms included in the study population rather than true frequency. However, Valderrabano et al. ${ }^{(25)}$ evaluated 463 indeterminate thyroid nodules (TNs) and concluded that there were no differences in the prevalence of malignancy between nodules with "low" (iso/hyperechoic) or "intermediate" (hypoechoic) suspicion patterns, concluding that hypoechogenicity alone did not seem to improve the risk stratification of indeterminate TNs.

There was a statistically significant difference between the two groups with benign and malignant nodules regarding the echogenic foci. This came in agreement with Dawoud and Dawoud ${ }^{(10)}$ who reported that presence of microcalcifications were found in $10(58.8 \%)$ of 17 malignant nodules, false positive results in 3 nodules, while no calcifications detected in $7(41.2 \%)$ malignant nodules. Nodule microcalcifications used alone as US patterns predictive of malignancy had sensitivity $58.82 \%$, specificity $93.02 \%$ and accuracy $83.33 \%$. Middleton et al. ${ }^{(19)}$ found that the risk of malignancy associated with peripheral calcifications, and punctate echogenic foci in solid nodules was $20.2 \%$ and $35 \%$ respectively. The results were also comparable results to those reported as we found that $11.1 \%$ of the malignant nodules had peripheral calcifications and $66.7 \%$ of the malignant nodules had punctate echogenic foci ${ }^{(7)}$. Our study also agreed with Reading et al. (26) who reported that macrocalcifications were found within both benign and malignant nodules yet more in the benign nodules.

In this study, there was a statistically significant difference between the two groups with benign and malignant nodules regarding the halo sign within the nodules. Halo sign was absent in $40 \%$ of the malignant nodules and present in $90 \%$ of the benign nodules. This came in accordance with Dawoud and Dawoud ${ }^{(\mathbf{1 0})}$ who reported that halo sign was absent in 16 benign nodules and 16 malignant nodules. Absent halo sign used alone as US patterns predictive of malignancy had sensitivity $94.12 \%$, specificity $62.79 \%$ and accuracy $71.67 \%$. Reiners $\boldsymbol{e t}$ al. ${ }^{(27)}$ found that at B-mode USG, isoechogenicity or hyperechogenicity and sonolucent halo were found in most of benign thyroid nodules.

Regarding the shape, thyroid cancer is associated with nodules with a ratio of anteroposterior to transverse diameter greater than one in the transverse view. Specificity ranges from 82 to $93 \%$; this means that it is a less suspicious nodule (21).

In this study, there was a statistically significant difference between the two groups with benign and malignant nodules regarding the vascularity shape. Taller than wider nodules were more common in the malignant lesions. In agreement with the results of this study, Azab et al. ${ }^{(7)}$ found that there was a statistically high significant increase in the incidence of taller than wider nodules in the malignant group than in the benign group with $\mathrm{P}$ value 0.001 . 
In this study, there was no statistically significant difference between the two groups with benign and malignant nodules regarding the margins of the nodules. Middleton et al. ${ }^{(19)}$ found that $12.9 \%$ of nodules with smooth margins and $44.7 \%$ of nodules with a lobulated or irregular border were malignant. Irregular and lobulated margins are suspicious for thyroid malignancy ${ }^{(28)}$. Azab et al. ${ }^{(7)}$ found that $11.1 \%$ of the malignant group nodules had smooth margins and $66.7 \%$ of the malignant group nodules had lobulated or irregular margins. It is important to assess extrathyroidal extension when evaluating thyroid malignancy. In our study, extrathyroidal extension was detected in two patients. In another study, irregular ill-defined margin was found in 17 nodules $(28.3 \%), 3$ (17.6\%) of them were benign and $14(82.4 \%)$ were malignant nodules, irregular margins used alone as US patterns predictive of malignancy had sensitivity $82.35 \%$, specificity $93.02 \%$ and accuracy $90 \%{ }^{(10)}$.

Some US features are described as suspicious for several studies, such as nodule hypoechogenicity, irregular margins, microcalcifications and taller-thanwide shape ${ }^{(23,29)}$. In this study, solid consistency, marked hypoechogenicity, taller than wider nodules, absent halo sign, peripheral and punctuate echogenic foci and smooth margins of the nodules were the ultrasonographic criteria associated with higher risk for malignancy. This is in accordance with Azab et al. ${ }^{(7)}$ who showed that there was a statistically highly significant increase in the incidence of solid thyroid nodules, very hypoechoic thyroid nodules, taller than wider nodules, nodules having lobulated or irregular margins, nodules having extrathyroidal extension, and thyroid nodules with punctate echogenic foci in the malignant group than in the benign group with $\mathrm{P}$ values <0.001, $0.001,0.001,<0.001,0.007$, and $<0.001$ respectively. Azab et al. ${ }^{(7)}$ found that $11.1 \%$ of the malignant group nodules had smooth margins and $66.7 \%$ of the malignant group nodules had lobulated or irregular margins. It is important to assess extrathyroidal extension when evaluating thyroid malignancy. In our study, extrathyroidal extension was detected in two patients.

\section{CONCLUSION}

The major ultrasound features seen associated with malignancy were microcalcifications, taller than wider shape of the nodule, hypoechoic and marked hypoechoic echopattern of the nodule, irregular borders and presence of suspicious cervical lymph nodes.

\section{REFERENCES}

1. Vanderpump M (2011): The epidemiology of thyroid disease, Br Med Bull., 99 (1): 39-51.
2. Harrison L, Hangiandreou O (2011): Physics tutorial for residents: Topics in US: B-mode US: Basic concepts and new technology - Hangiandreou. Radiographics, 23(4): 1019-1033.

3. Salahudin $\mathbf{T}$ (2016): Thyroid computed tomography imaging: pictorial review of variable pathologies. Insights into Imaging. Insights Into Imaging, 7 (4): 601-617.

4. Durante C, Grani G, Lamartina L et al. (2018): The diagnosis and management of thyroid nodules: a review. JAMA., 319(9):914-24.

5. Durmaz M, Akyürek N, Kara T et al. (2019): Quantitative assessment of thyroid gland vascularization with vascularization index using color superb microvascular imaging in pediatric patients with hashimoto thyroiditis. Ultrasound Quarterly, 35(3):2819.

6. Mosconi C, Crocetti L, Bruno A et al. (2020): Scar pregnancy and extrauterine implants. Seminars in ultrasound, $\quad \mathrm{CT}$ and https://www.sciencedirect.com/science/article/abs/pii/S0887 21712030086X

7. Azab E, Abdelrahman A, Ibrahim M (2019): A practical trial to use Thyroid Imaging Reporting and Data System (TI-RADS) in differentiation between benign and malignant thyroid nodules. Egyptian Journal of Radiology and Nuclear Medicine, 50(1): 17-22.

8. Horvath E, Silva C, Majlis S et al. (2017): Prospective validation of the ultrasound based TIRADS (Thyroid Imaging Reporting and Data System) classification: results in surgically resected thyroid nodules. European Radiology, 27(6): 2619-2628.

9. Ashamallah G, EL-Adalany M (2016). Risk for malignancy of thyroid nodules: Comparative study between TIRADS and US based classification system. The Egyptian Journal of Radiology and Nuclear Medicine, 47(4): 1373-1384.

10. Dawoud M, Dawoud R (2017): Added value of strain elastosonography in prediction of malignancy in solitary thyroid nodule. The Egyptian Journal of Radiology and Nuclear Medicine, 48(4): 905-912.

11. Batawil N, Alkordy $T$ (2014): Ultrasonographic features associated with malignancy in cytologically indeterminate thyroid nodules. European Journal of Surgical Oncology (EJSO), 40(2): 182-186.

12. Barbosa T, Junior C, Graf $H$ et al. (2019): ACR TIRADS and ATA US scores are helpful for the management of thyroid nodules with indeterminate cytology. BMC Endocrine Disorders, 19(1): 112-119.

13. Paschke R, Hegedüs L, Alexander E et al. (2011): Thyroid nodule guidelines: agreement, disagreement and need for future research. Nature Reviews Endocrinology, 7(6): 354-361.

14. Periakaruppan G, Seshadri K, Krishna G et al. (2018): Correlation between ultrasound-based TIRADS and Bethesda system for reporting thyroidcytopathology: 2-year experience at a tertiary care center in India. Indian Journal of Endocrinology and Metabolism, 22(5): 651-56. 
15. Moifo B, Takoeta E, Tambe J et al. (2013): Reliability of thyroid imaging reporting and data system (TIRADS) classification in differentiating benign from malignant thyroid nodules. Open J Radiol., 3(3): 103-7.

16. Chandramohan A, Khurana A, Pushpa B et al. (2016): Is TIRADS a practical and accurate system for use in daily clinical practice?. The Indian Journal of Radiology \& Imaging, 26(1): 145-52.

17. Srinivas $M$, Amogh V, Gautam $M$ et al. (2016): A prospective study to evaluate the reliability of thyroid imaging reporting and data system in differentiation between benign and malignant thyroid lesions. Journal of Clinical Imaging Science, 6(1):5-10.

18. Tessler F, Middleton W, Grant E et al. (2017): ACR thyroid imaging, reporting and data system (TI-RADS): white paper of the ACR TI-RADS committee. Journal of the American College of Radiology, 14(5): 587-595.

19. Middleton W, Teefey S, Reading C et al. (2017): Multiinstitutional analysis of thyroid nodule risk stratification using the American College of Radiology Thyroid Imaging Reporting and Data System. American Journal of Roentgenology, 208(6): 13311341.

20. Russ G, Royer B, Bigorgne C et al. (2013): Prospective evaluation of thyroid imaging reporting and data system on 4550 nodules with and without elastography. Eur J Endocrinol., 168(5): 649-55.

21. Ahn S, Kim E, Kang D et al. (2010): Biopsy of thyroid nodules: comparison of three sets of guidelines. American Journal of Roentgenology, 194(1): 31-37.

22. Kovacevic O, Smetana-Škurla M (2007). Sonographic diagnosis of thyroid nodules: Correlation with the results of sonographically guided fine-needle aspiration biopsy. Journal of Clinical Ultrasound, 35(2): 63-67.
23. Kwak J, Han K, Yoon J et al. (2011): Thyroid imaging reporting and data system for US features of nodules: a step in establishing better stratification of cancer risk. Radiology, 260(3):892-9.

24. Na D, Kim J, Kim D et al. (2016): Thyroid nodules with minimal cystic changes have a low risk of malignancy. Ultrasonography, 35(2):153-59.

25. Valderrabano $\mathbf{P}$, Khazai L, Thompson $\mathbf{Z}$ et al. (2018): Association of tumor size with histologic and clinical outcomes among patients with cytologically indeterminate thyroid nodules. JAMA OtolaryngologyHead \& Neck Surgery, 144(9): 788-795.

26. Reading C, Charboneau J, Hay I et al. (2005): Sonography of thyroid nodules: a "classic pattern" diagnostic approach. Ultrasound Quarterly, 21(3): 157165.

27. Reiners C, Wegscheider K, Schicha H et al. (2004): Prevalence of thyroid disorders in the working population of Germany: ultrasonography screening in 96,278 unselected employees. Thyroid, 14(11): 926932.

28. Ito Y, Kobayashi K, Tomoda C et al. (2005): Illdefined edge on ultrasonographic examination can be a marker of aggressive characteristic of papillary thyroid microcarcinoma. World Journal of Surgery, 29(8): 1007-1011.

29. Grant E, Tessler F, Hoang J et al. (2015): Thyroid ultrasound reporting lexicon: white paper of the ACR thyroid imaging, reporting and data system (TIRADS) committee. Journal of the American College of Radiology, $\quad 12(12)$ : $1272-1279$. 